\title{
Trust Meta-Policies for Flexible and Dynamic Policy Based Trust Management
}

\author{
Karl Quinn, David Lewis, Declan O'Sullivan, Vincent P. Wade \\ Knowledge and Data Engineering Group, Trinity College Dublin, Ireland. \\ $\{$ Karl.Quinn|Dave.Lewis|Declan.Osullivan|Vincent.Wade\}@cs.tcd.ie
}

\begin{abstract}
It is possible to impose the will of the user or administrator through the specification of policies. These policies reflect the users or administrators goals; however the context in which these goals operate can vary greatly. This paper builds on our previous work where we demonstrated the creation and use of policies that had trust conditions embedded. The work reported on here exposes these trust conditions explicitly as trust meta-policies.
\end{abstract}

\section{Introduction}

The need to use and manage trust is becoming increasingly important in policy research. However, trust is a very subjective concept and therefore requires more personalised approaches that can reflect trusts subjective nature. The challenge is to provide such personalised models that can operate in conjunction with policy to provide a flexible and dynamic management system.

There are a myriad of meanings or definitions for trust $[1,2,3]$, and a wealth of trust management systems $[4,5,6]$. Trust can be used to guide decision making in order to reflect the wishes of the administrator. However, in [7], it is stated that trust is bad for security as trust itself is an overloaded term with intertwined meanings. Our approach to trust $[8,9$, $10,11]$ utilises a rich, semantic, model of trust that was designed and developed to support such different and intertwined ideas about trust. This approach uses personalised models of trust that are part of an overall trust management system. The trust management system can operate over each of the personalised models of trust to provide personalised trust recommendations.

In [10], we illustrated how a Community Based Policy Management (CBPM) system and a Trust Based Access Control (TBAC) system can provide flexible, relationship-driven policy management. In that research the access policies themselves were based solely on trust information. Community members were therefore granted access so long as they met the trust requirements that was the policy.

In this paper we illustrate how we have advanced the research presented in [8], [9], and [10] where policies had trust conditions embedded through the introduction of trust meta-policies. Trust meta-policies expose the trust conditions explicitly.

\section{Trust Model Approach}

Trust meta-policies are based on a model of trust that can be personalised on a per user basis and specialised on a per domain basis. As per figure 1, we designed and developed an upper ontology that consists of a set of trust concepts that can be related to each other by using an accompanying set of relationships.

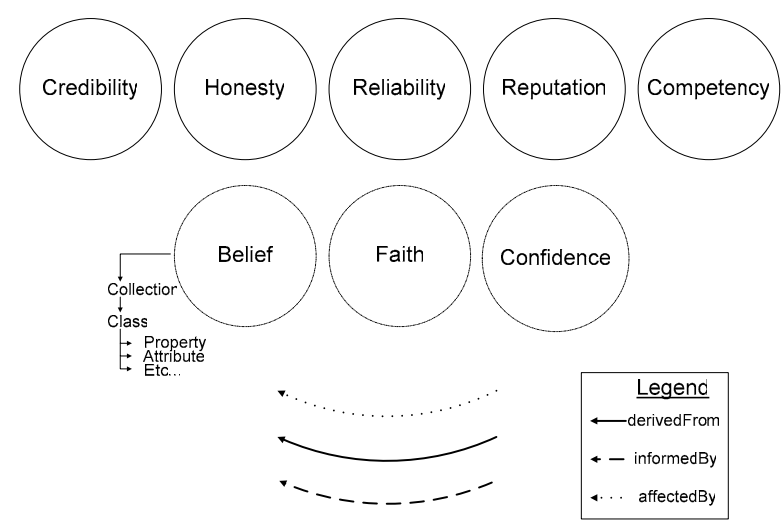

Fig 1 Upper Ontology

The upper ontology is used to create a personalised model of trust as per figure 2 . In this way it is possible for an individual to generate a personalised model of trust specific to their idea of trust. Note that it is also possible to generate a personalised model of trust for a community or business. 
Please see $[9,10]$ for further information about the upper ontology, personalised models of trust, and specialising the trust model towards multiple domains.

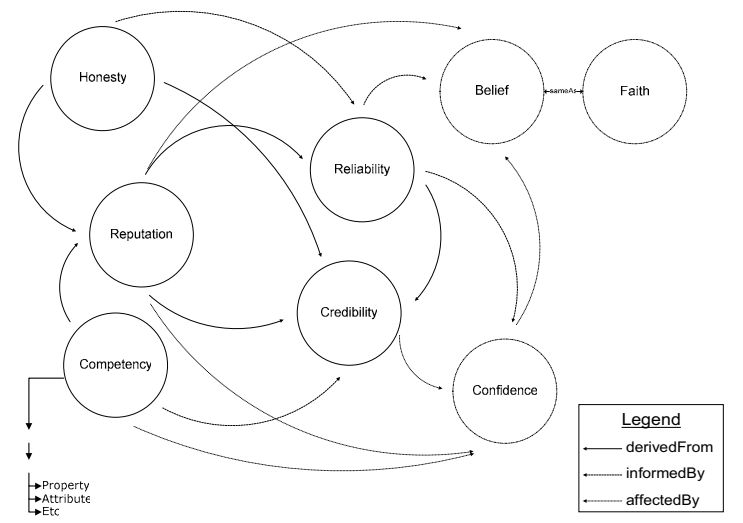

Fig 2 Personalised Model of Trust

The trust management system that the upper and personalised models of trust operates in, empowers a user to annotate other users with trust data in relation to each trust concept. This information can then be shared on a collaborative basis in decentralised peer to peer operations. This shared trust data is used in conjunction with the user's personalised model of trust to calculate personalised trust recommendations.

\section{Trust Meta-Policies}

In [10], the policies that regulated access control were based on trust conditions and other non-trust conditions. Each of these meta-policies has a trust condition that states a minimum overall trust threshold value of either very low, low, high, or very high. This overall trust threshold value is calculated on a per user basis. In a trust meta-policy events are based on risk and conditions are based on trust information. Through experimentation we have confirmed an important correlation between risk and trust. This correlation states that as the level of risk increases so too does the reliance on trust increase. The benefits of a trust metapolicy approach in comparison to an integrated approach are numerous. Firstly, the trust meta-policies and standard policies can be managed independently and used independently or used in combination. This provides a clear separation of concerns. Secondly, the trust meta-policy empowers the user to specify events as low, medium, or high risk. Standard policies can be naturally associated with these risk levels. The combination of standard policy and trust meta-policy provides a flexible and dynamic policy based trust management approach.

For example, take a scenario where a user has a standard policy that is used in relation to online auctions. It simply states that a bid action can only be made on the condition that the seller will deliver to Ireland. This policy is presented in figure 3 and is based in the XACML 2.0 specification [12]. However, the policy is presented in a simplified form.

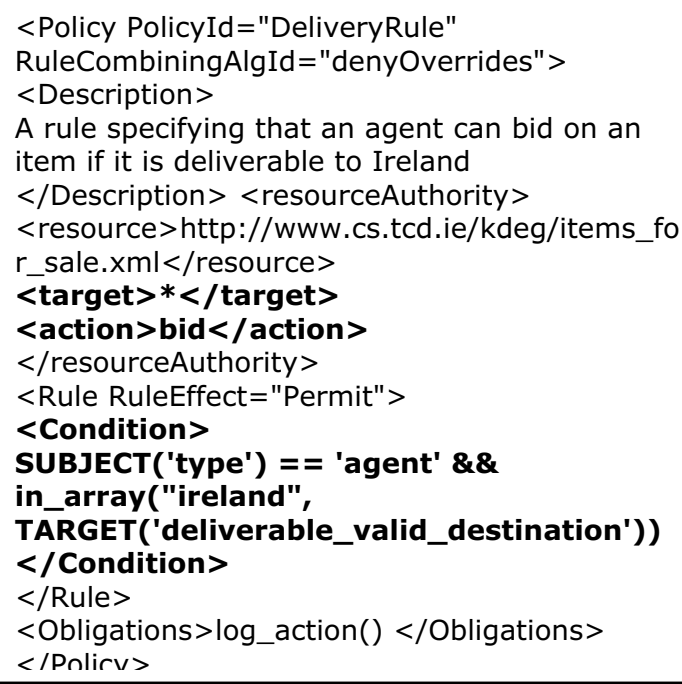

Fig 3 Personalised Model of Trust

Our user, a buyer, has set up an agent to make bids on high volume, repeat items, such as office stationary in order to maximise her efficiency. She has also created a set of trust meta-policies for low, medium, and high-risk contexts as per figure 4. A low risk context is considered a bid up to $\$ 10$ and our buyer requires the seller to have at least a low overall trust threshold value. At medium risk, up to $\$ 100$, the seller is required to have a high overall trust threshold value. The high risk policy, up to $\$ 1000$, requires the seller to have a very high overall trust threshold value. The original bidding policy works in conjunction with the meta-policies that provide guidance as context changes; as risk increases.

\begin{tabular}{|c|c|c|c|}
\hline & $\begin{array}{c}\text { Low Risk } \\
(\mathbf{\$ 1 0 )}\end{array}$ & $\begin{array}{c}\text { Medium Risk } \\
\mathbf{( \$ 1 0 0 )}\end{array}$ & $\begin{array}{c}\text { High Risk } \\
\mathbf{( \$ 1 0 0 0 )}\end{array}$ \\
\hline $\begin{array}{c}\text { Required } \\
\text { Trust } \\
\text { Value }\end{array}$ & Low & High & Very High \\
\hline
\end{tabular}

Fig 4 Example Buyer's Meta-Policy

In operation, the agent will interact with the auction system and other agents on the buyer's behalf. In the event that the buyer's agent would like to bid on an item worth $\$ 5$ the agent will ensure that delivery can be made to Ireland and then consult the trust metapolicy set for advice. The trust framework will then seek evidence from other buyer and seller agents 
willing to share trust information with respect to the seller in this particular auction. The trust data returned to the buyer's agent will be process in conjunction with the buyer personalised model of trust. This calculation will render a final trust value for the seller that is either; very low, low, high or very high trust. In the event where the bid price is $\$ 5$ and the system has calculated that the seller has a high trust value. According to the buyer's trust meta-policy set, figure 3 , the seller's trust value exceeds the minimum requirements set out by the buyer for a low risk context. Therefore, the trust recommendation will be positive. If the bid price was $\$ 75$ the recommendation would not have changed as the seller would have still met the trust requirements of the user. However, at $\$ 300$ the buyer's agent would have received a negative recommendation and the bid would not have taken place.

\section{Evaluation of Meta-Policy Approach}

We created a survey of opinion through a questionnaire. We carried out this survey to evaluate the trust meta-policy approach. In this questionnaire each subject first provided information specific to the trust concepts that enabled the generation of a personalised model of trust. The subject then created a set of trust meta-polices for low, medium, and high risk contexts. Four specific type of people were annotated with trust data; a family member, a work colleague, a friend of a friend, and a stranger. The questionnaire had four actions that a user could allow; the use of their; pencil, mobile, laptop, and bank Personal Identification Number (PIN). The subjects specified which meta-policy each of four actions had associated with it, e.g. using laptop is medium risk. Finally, they answered a set of person-action questions that related each annotated person to each action, e.g. 'would you allow a work colleague to use your laptop?'

The survey enabled us to collect enough information to automatically generate a personalised model of trust for each subject. This personalised model was used to drive calculations for each action relative to annotated trust information. These calculations were used to predict the outcome of the subject's person-action question set. This allowed for a comparison to be made between the subject's personaction answer set and the predicted person-action set.

As per the first column, 'allowed action', in figure 5 the analysis between the subject's set and the calculated set found a $78 \%$ success rate in correctly reflecting what the subject stated in relation to actions that were allowed. The incorrect $22 \%$ can be attributed to the stranger, family member, FOAF, or colleague not meeting the minimum trust requirements as set out by the user. As can be seen from the second column, 'disallowed actions', in figure 5 the analysis found a $100 \%$ success rate in correctly reflecting what the user stated in relation to actions that were not allowed. In these situations a user stated that an action should not take place, as did the system.

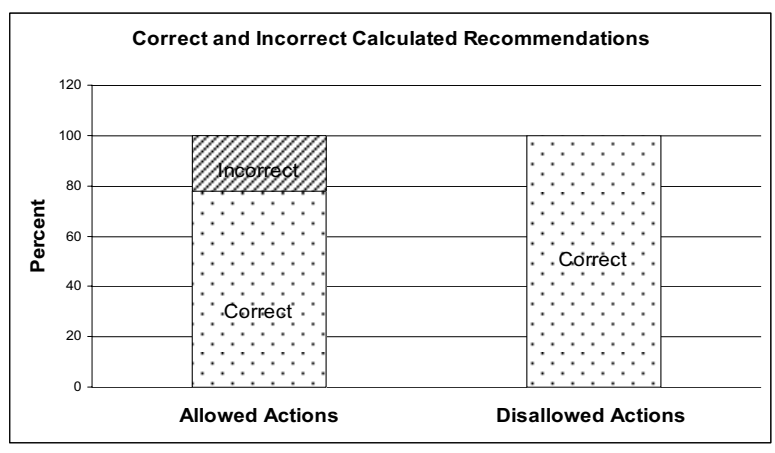

Fig 5 Analysis Graph

Using this analysis data in relation to the online auction scenario we can postulate the following;

A. In the event where a user would state that she would allow a bid to be made on an item, the trust system would reflect this accurately $78 \%$ of the time.

B. In the event where a user would state that she would not allow a bid to be made on an item, the trust system would reflect this accurately $100 \%$ of the time.

The analysis indicated that the vast majority of the incorrect recommendations were within one degree of the required minimum trust level. For example, a family member was denied the knowledge of the subject's bank PIN when they had an overall trust value of high and where a very high trust value was required. We suggest a polling system that can ask the subject for input in cases where the difference between trust value and required trust is one degree or less.

Although the system is not entirely accurate in identifying situations where the user would allow an action it is very accurate in identifying situations in which the user would not allow an action to take place. Prompting the user for a definitive course of action in cases where the required trust is below one degree can help the system learn and reduce the overall level of user interaction in online auctions. The safety net that the negative accuracy provides is a powerful and useful feature of the system. Our agent can seek negative recommendation accurately. Where a user is not present to provide definitive actions the agent would derive its guidance mainly from the policy 
associated with the action and then carry out actions unless the trust management system expressly recommends not to.

We also have a set of user responses to questions asked in relation to actions and annotated people, the person-answer set. Through analysis we have noticed that either the subject has created meta-policies that are too stringent or the user in question does not have sufficient trust information to allow the action to take place. This set of answers can be used to train the calculation algorithm to provide more accurate reflections of what the subject requires.

\section{Conclusions and Further Work}

The trust meta-policy system that we have outlined has been shown to provide very accurate recommendation in relation to actions that should not be taken. However, we acknowledge that the initial sample size of nine subjects is quite small. When combined with interactions from the user, recommendations that are based on actions that may be taken can also be accurately provided. Combining the trust meta-policy approach with agents provides guidance for the agents to ensure that actions which are not allowed are not taken.

The future research work will investigate the possibility of improving the accuracy of the calculated predictions. This might be achieved by enabling the system to learn through user feedback. Future work in relation to generating models of trust, annotating other users with trust information, and creating policies to empower Trust Based Access Control will concentrate on evaluating results and improving the mechanisms surrounding the trust management system.

\section{Acknowledgements}

This work is partially supported by the Irish Higher Education Authority under the M-Zones programme. Karl Quinn's work receives a scholarship from IRCSET.

\section{References}

[McKnight] McKnight, H.D., Chervany, N.L., 'The Meanings of Trust; Technical Report 94-04, Carlson School of Management, University of Minnesota', 1996.

[1] Golbeck, J., Hendler, J., Parsia, B. 'Trust Networks on the Semantic Web', 12th International Web Conference (WWW03),Budapest, Hungary, May 2003.
[2] Shadbolt, N., 'A Matter of Trust', IEEE Intelligent Systems, pp. 2-3 January/February 2002.

[3] Grandison, T., Sloman, M., 'A Survey of Trust in Internet Applications', IEEE Communications Surveys, 3, pp. 2-16, Fourth Quarter 2000.

[4] Grandison, T., Sloman, M., 'SULTAN - A Language for Trust Specification and Analysis', Proceedings of the 8th Annual Workshop HP OpenView University Association (HP-OVUA), Berlin, Germany, June 24-27, 2001.

[5] Levien, R., Aiken, A.,'Attack resistant trust metrics for public key certification.', 7th USENIX Security Symposium, San Antonio, Texas, January 1998.

[6] Gil, Y., Ratnakar, V., 'Trusting Information Sources One Citizen at a Time.', Proceedings of the First International Semantic Web Conference (ISWC), Sardinia, Italy, June 2002.

[7] Gollmann, D., 'Why Trust is Bad for Security', Keynote Speech, IEEE 6th International Workshop on Policies for Distributed Systems and Networks (POLICY 2005), Stockholm, Sweden, 6-8 June, 2005.

[8] Feeney, K., Lewis, D., Wade, V. "Policy-based Management for Internet Communities", in proc of 5 th IEEE International Workshop on Policies and Distributed Systems and Networks, IEEE, 2004

[9] Quinn, K., O'Sullivan, D., Lewis, D., Brennan, R., Wade, V.P., 'deepTrust Management Application for Discovery, Selection, and Composition of Trustworthy Services.' Proceedings of IDIP/IEEE 9th International Symposium on Integrated Network Management (IM 2005), Nice, France, May 2005.

[10] Feeney, K., Quinn, K., O'Sullivan, D., Lewis, Wade, V.P., 'Relationship-Driven Policy Engineering for Autonomic Organisations', IEEE 6th International Workshop on Policies for Distributed Systems and Networks, Stockholm, Sweden, 6-8 June, 2005.

[11] Quinn, K., Kenny, A., Feeney, K., Lewis, D., O'Sullivan, D., Wade, V.P., 'Flexible Relationshipdriven Management in the Decentralisation of Collaborative, Context Aware Systems', 10th IEEE/IFIP Network Operations and Management Symposium, Vancouver, Canada, April 3-7, 2006.

[12]http://www.oasis-

open.org/specs/index.php\#xacmlv2.0 